\title{
Choice of parameters of oscillatory actions in the molding of reinforced concrete products
}

\author{
Vitaly Vasiliev ${ }^{1, *}$ \\ ${ }^{1}$ Moscow State University of Civil Engineering, Yaroslavskoe shosse, 26, Moscow, 129337, Russia
}

\begin{abstract}
The process of propagation of a wave packet in the concrete mixture during the formation of reinforced concrete products by harmonic and shock-vibrational actions was considered. It was shown that the displacement of the layers of the concrete mixture during the propagation of the shock wave is directed against the direction of wave propagation, providing a sealing effect. On the basis of experimental studies, practical recommendations are given for determining the rational thickness of the compacted concrete mixture, depending on the frequency of oscillations.
\end{abstract}

\section{Introduction}

At present, with large volumes of prefabricated reinforced concrete production, many largescale reinforced concrete products of high factory readiness are produced, to which high demands are imposed on uniformity and strength [1-5]. Traditional (standard) molding conditions do not fully provide high quality and corresponding technical properties of concrete throughout the volume of products due to rapid attenuation of oscillations in the concrete mixture. This disadvantage is compensated to some extent by the use of the lowfrequency shock-vibration molding technology [6-11].

Under symmetrical molding conditions, complex phenomena of the interaction of wave fronts arising in the column of the concrete mixture have been sufficiently studied in the works of various authors [12-16]. It is of interest to investigate the process of propagation of a wave packet in the concrete mixture with a shock-vibration action on the molded product.

\section{Research methods}

Let us consider the propagation of longitudinal oscillations of a column of the concrete mixture of height $L$, when the lower end is affected by harmonious forcing:

$$
U(y, t)=A \sin \omega t
$$

where $A$ - amplitude of mold bottom plate oscillations;

$U(y, t)$ - displacement.

\footnotetext{
* Corresponding author: vasilievvg@yandex.ru
} 
To simplify the calculations, we assume that there is no attenuation in the column of compacted concrete (which will only affect the oscillation amplitude value of the particles of steady-state oscillations).

The differential equation (in partial derivatives) of a given boundary-value problem has the form:

$$
\frac{\partial^{2} U}{\partial t^{2}}=C_{0}^{2} \frac{\partial^{2} U}{\partial y^{2}} \quad 0<y<L ; \quad 0<t<\infty,
$$

under the boundary conditions:

$$
U(L, t)=0 ; \quad U(0, t)=A \sin \omega t,
$$

and the initial conditions:

$$
U(y, 0)=\frac{\partial U}{\partial t}(y, 0)=0 ; \quad 0<y<L
$$

where $C_{0}$ - wave packet propagation speed

The solution of equation (1) with $\omega \neq \frac{n \pi A}{L}$, where $n=1,2,3 \ldots i$ has the form:

$$
U(y, t)=A \frac{\sin \frac{\omega y}{C_{0}}}{\sin \frac{\omega}{C_{0}} L} \sin \omega t+\sum_{n=1}^{\infty} b_{n} \sin \frac{n \pi y}{L} \sin \frac{n \pi C_{0} t}{L}
$$

where $b_{n}=-\frac{2 A \omega}{n \pi C_{0}} \int_{0}^{2} \frac{\sin \frac{\omega}{C_{0}} y}{\sin \frac{\omega}{C_{0}} L} \sin \frac{n \pi y}{L} \partial y ; \quad n=1,2,3 \ldots i$.

From the analysis of formula (5) it follows that the oscillations of the particles in the mixture occur with the same frequency equal to the frequency of the oscillatory action.

Now let us consider the oscillations of the column of height L, on the bottom plate of which a shock (impulse) action is produced, the value of the impulse of which is equal to $Y$. Just as in the first case, the medium is considered without attenuation.

The statement of the boundary value problem has the form:

$$
\frac{\partial^{2} U}{\partial t^{2}}=C_{0}^{2} \frac{\partial^{2} U}{\partial y^{2}} ; \quad 0<y<L ; \quad 0<t<\infty,
$$

with the following boundary and initial conditions:

$$
U(y, 0)=0 ; \quad \frac{\partial U}{\partial t}(y, 0)=\left\{\begin{array}{c}
0 \\
-\frac{\gamma}{\delta \rho} ; \quad 0<y<L-\delta ; \quad L-\delta<y \leq L .
\end{array}\right.
$$

Here with $\delta$ tends to 0 (impulse duration is minimal), where $\rho$ - density of the concrete mixture.

The solution has the form: 


$$
U(y, t)=-\frac{\gamma}{\rho L} t-\frac{2 \gamma}{C_{0} \pi \rho} \sum_{k=1}^{\infty} \frac{(-1)^{k}}{k} \cos \frac{k \pi y}{L}
$$

It is easy to see that the first term of formula (8) increases formally with increasing time.

Let us consider the value relation:

$$
\frac{\gamma}{\rho L} t \quad \text { and } \quad \frac{2 \gamma}{\pi C_{0} \rho}
$$

Considering that $L=C o t$, under this condition, the first oscillation form propagates in the mixture column according to the theory of B. Gusev [6].

Substituting (9) into the first term of formula (8), we obtain $\frac{\gamma}{\rho C_{0}}$. It follows that the first displacement component exceeds the second one in modulus, the value of which decreases even more when there are harmonic factors $\cos \frac{k \pi y}{L}$ and $\sin \frac{k \pi C_{0} t}{L}$.

The minus sign (-) before the first displacement component $U(y, t)$ in formula (8) indicates that the displacement of the layers during the shock wave propagation are directed only downward, against the direction of the wave propagation, and do not decrease in value as they approach the column surface. Subsequent shock impulses can only increase (without or with low attenuation) displacement of the layers downward.

The propagation of oscillation disturbances in the concrete mixture is a wave process that is characterized by the wave velocity and length and is related to the viscoelastic properties of the medium. When forming the high layers of the concrete mixture, one should take into account the ratio of the wave length and the product height in terms of creating favorable conditions for uniform compaction of all layers of the mixture in height $[6 ; 16]$.

The optimal pulse repetition rate $f_{\text {opt }}$ is determined from the condition of minimum energy costs to obtain a sealing effect: the height of the column of the concrete mixture should be equal to the wave length at a given propagation speed:

$$
f_{\text {opt }}=\frac{C_{0}}{L}
$$

So, the higher the height of the compacted column of the concrete mixture, the smaller the frequency. In this case (in the case of impact on the mixture), the displacements of the layers of the mixture have the same sign and are directed only to the bottom plate, thereby the "pressing effect" is carried out.

With the harmonic action on the concrete mixture, as follows from formula (5), the layer of the mixture vibrates near the equilibrium position, its displacements change sign, thus the above-mentioned "pressing effect" is absent.

The distribution of the obtained solutions to real concrete mixtures, which are multiphase multicomponent media, will be more correct if we take into account the degree of influence of the shape and spectrum of the shock-vibration impulse on the behavior of the compacted medium [17].

To confirm the obtained results, the experimental studies of the wave process that occurs during the spread of vibrations in concrete mixture when forming the reinforced concrete products of different heights have been undertaken. The speed of propagation of the wave packet was determined by the method of holographic stroboscope $[7,18]$. 
Studies were carried out on concrete mixtures in a wide range of workability: from a slump of 11-12 cm to hard mixtures characterized by the hardness of $60 \mathrm{~s}$ by a technical viscometer. The mixtures were compacted by harmonic and shock-vibration actions with different oscillation parameters. The obtained values of the wave propagation velocity for different compositions and action conditions are given in Table 1.

Table 1. Velocity of the wave packet propagation, $\mathrm{m} / \mathrm{s}$, in the concrete mixture at various frequencies of oscillations of the working.

\begin{tabular}{|c|c|c|c|}
\hline $\begin{array}{c}\text { Frequency of } \\
\text { oscillations of a } \\
\text { working } \\
\text { element, } \mathrm{Hz}\end{array}$ & $\begin{array}{c}|c| \\
\text { Slump } \\
11 \mathrm{~cm}\end{array}$ & $\begin{array}{c}\text { Hardness of the } \\
\text { concrete } 60 \mathrm{~s}\end{array}$ & \\
\hline 15 & $33 \ldots 46$ & $34 \ldots 45$ & $30 \ldots 48$ \\
\hline 25 & $50 \ldots 64$ & $37 \ldots 54$ & $35 \ldots 55$ \\
\hline 50 & $58 \ldots 72$ & $53 \ldots 65$ & $59 \ldots 75$ \\
\hline 100 & $85 \ldots 90$ & $75 \ldots 85$ & $80 \ldots 105$ \\
\hline
\end{tabular}

Analysis of the data of Table 1 showed that the range of variation in the propagation velocities of the wave front in concrete mixtures in the case of using harmonic and shockvibration actions at fixed oscillation frequencies of the working element is practically the same.

Competent visual comparison of the nature of the wave front propagation under symmetrical and asymmetric actions on the mixture carried out in Refs $[7,18]$ showed, that in the case of shock-vibration actions, a clear front of the wave packet was not seen at a distance up to 300-350 mm from the place of vibration application. At a height of 355-400 $\mathrm{mm}$, two harmonics were observed: the main one with a frequency of $25 \mathrm{~Hz}$ and the second one - $30 \mathrm{~Hz}$. At heights above $450 \mathrm{~mm}$, the main carrier harmonic was recorded, propagating at a speed of $45-60 \mathrm{~m} / \mathrm{s}$. When using symmetrical oscillations, the wave front had a clear harmonic picture.

In the process of forming large-size reinforced concrete products, it is important to know the value of the propagation velocity of the oscillations along the thickness of the layers to be compacted. In this case, it is possible to choose a rational form of oscillations, in which uniform compaction of the mixture throughout the volume of the large-sized product is ensured.

Depending on the ratio of the wave length and the height of the molded product, zones with the same and oppositely directed oscillations appear in the concrete mixture, which affects the degree of uniformity of the compaction along the height of the compacted mixture.

It is known $[6,16,19,20]$ that the best conditions for uniform and high-quality compaction of concrete mixtures in the height of the product are created when the column height is $1 / 4$ or $3 / 4$ of the wave length, which can be calculated from the used frequency of oscillations and group velocity of their propagation.

Thus, from the known values of the velocities of the wave packet propagation, it is possible to determine the limiting compaction thicknesses of concrete mixtures for any oscillation form.

Table 2 shows rational thicknesses of the concrete mixture compaction, depending on the frequency of oscillations.

From Table 2 it can be seen that, with the increasing frequency of the oscillations of the working element, the zone of effective compaction of the concrete mixture decreases substantially, making an average $30 \mathrm{~cm}$ for a standard frequency of $50 \mathrm{~Hz}$. 
Table 2. Rational thicknesses of the concrete mixture of various workability, depending on the frequency of oscillations.

\begin{tabular}{|c|c|c|}
\hline $\begin{array}{c}\text { Frequency of } \\
\text { oscillations of a } \\
\text { working } \\
\text { element, } \mathrm{Hz}\end{array}$ & $\begin{array}{c}\text { Zone of effective compaction of the } \\
\text { concrete mixture, cm }\end{array}$ \\
\cline { 2 - 3 } & Slump 11 cm & Hardness $15 \mathrm{~s}$ \\
\hline 15 & $70 \ldots 80$ & $55 \ldots 70$ \\
\hline 25 & $55 \ldots 65$ & $40 \ldots 55$ \\
\hline 50 & $30 \ldots 35$ & $25 \ldots 30$ \\
\hline 100 & $8 \ldots 10$ & $6 \ldots 8$ \\
\hline
\end{tabular}

\section{Conclusions}

A number of studies of wave process that occurs during the spread of vibrations in concrete mixtures of different heights indicate that forming the large-scale reinforced concrete products and constructions should be made by the use of the low-frequency shock-vibration molding technology. Meanwhile, knowing the velocity of propagation of wave front and the vibration frequency, it is possible to define the limiting thicknesses of the effective and equal compaction of the concrete mixtures.

\section{References}

1. S. Pulyaev, M. Stepanov, B. Kaitukov and others Mechanical equipment and technological complexes: manual (M.: MGSU, 2015)

2. A. Borshchevsky, A. Ilyin Mechanical equipment of enterprises for the production of building materials and products (Moscow, Alliance, 2009)

3. E. Chabutkin, A. Barulev, J. Mech. of const., 7 (2017)

4. B. Gusev, E. Axelrod, A. Zvezdov and others Manual on the technology of molding of reinforced concrete products (Moscow, Stroiizdat, 1988)

5. Kh. Muzakayev, A. Ertuev, B. Ladaria and others, J. Econ. and Entreprenp., 96, 7 (2018)

6. B. Gusev Theory and practice of compaction of concrete mixtures in low-frequency modes of vibration (D.Sc. thesis, Moscow, 1977)

7. E. Zinoviev Forming of large-size ferro-concrete products with the use of controlled modes of vibration compaction (PhD work, M., 1989)

8. V. Vasiliev, J. Mech. of const., 11 (2015)

9. A. F. Itkin Vibration machines for molding concrete products (Kiev, MP Lesya, 2009)

10. V. Bogdanov, R. Sharapov, Yu. and others The fundamentals of the calculation of machines and equipment of enterprises of construction materials and products.: textbook (Stary Oskol, TNT, 2013)

11. A. Maslov, B. Zhanar, J. Modern scien. res. and innov, 68, 12 (2016)

12. B. Gusev, V. Zazimko Vibration technology of concrete (Kiev, Budivelnik, 1991)

13. O. Savinov, E. Lavrinovich Vibration technology of compaction and formation of concrete mixtures (M., Stroiizdat, 1986)

14. I. Nazarenko Highly efficient vibroforming machines (Kiev, Vishcha shkola (1988).

15. S. Gobozov, V. Tibilov. I. Khugaev, J. Sciences of Europe, 21-1 (21) (2017)

16. A. Fayvusovich, B. Gusev Technological mechanics of vibrating concrete mixtures (M., Scientific world, 2002)

17. R. Sharapov, V. Vasiliev, MATEC Web of Conf., 117 (2017) 
18. E. Zinoviev, V. Vasiliev, E. Parfenov, M., Dep. VINITI, Households and buildings, 745-B99 (1999)

19. A. Petrov, B. Lishansky, E. Rafales-Lamarck, Engineering Phys. J. Minsk, Sc. and Tech., Ac. of Sc. of the BSSR, 37, 4 (1979)

20. I. Sivak, Yu. Chovnyuk, V. Kravchuk, Bul. of Kherson Nat. Tech. Un., 58, 3 (2016) 